\title{
Airway obstruction by extrinsic tracheal compression during spinal surgery under prone position -A case report-
}

\author{
Rak Min Choi, Jin Sun Yoon, Jae Hoon Noh, Kyoung Oh Kang, Sang Wook Ryu, Hee Jung Jun, and \\ Sam Soon Cho \\ Department of Anesthesiology and Pain Medicine, Seoul Veterans Hospital, Seoul, Korea
}

Tracheal compression by vascular anomalies in adults is uncommon and most related reports are of children. A 79-year-old woman without any respiratory history underwent a lumbar spine surgery under general anesthesia. She suddenly developed airway obstruction after a position change from supine to prone. A fiberoptic bronchoscopy showed the obstruction of endotracheal tube. The obstruction was relieved after we changed the depth of endotracheal tube and supported the patient's neck with a cotton roll. The surgery ended without any other event and the patient recovered safely. A computed tomography revealed the rightward tracheal deviation and tortuous innominate artery contact with trachea. The patient didn't manifest any respiratory related symptoms during postoperative period, and she was discharged without any treatment. (Korean J Anesthesiol 2010; 59: S45-S48)

Key Words: Fiberoptic bronchoscopy, Prone position, Tracheal compression, Vascular anomaly.

\begin{abstract}
Airway can be compressed by vascular malformations such as innominate artery anomaly or right aortic arch. As such vascular malformation occurs mainly in inherent, it is usually detected right after birth or in infants but rare in adults $[1,2]$. When an infant or a child experiences repeatedly respiratory problems with an unknown origin, such as upper airway obstruction, wheezing and stridor, dysphasia, or obstructive apnea, or when an adult has a history of the above symptoms that have been repeated, it is likely that the patient's airway is mechanically obstructed. Especially a special attention should be paid to
\end{abstract}

the type of vascular anomalies and a possibility of having heart disease or any intrinsic tracheobronchial tree lesion as anesthesia varies according to them. Authors report a case with literature review where a patient with an unknown vascular malformation has a sudden respiratory problem after changing a position from supine to prone for a lumbar spine surgery. The patient could finish the operation safely after adjusting the depth of an endotracheal tube by confirming obstruction of the tube under fiberoptic bronchoscopy and supporting the patient's neck with a cotton roll.

Received: March 10, 2010. Revised: 1st, March 20, 2010; 2nd, April 5, 2010. Accepted: April 14, 2010.

Corresponding author: Jin Sun Yoon, M.D., Department of Anesthesiology and Pain Medicine, Seoul Veterans Hospital, 6-2, Dunchon-dong, Gangdong-gu, Seoul 134-060, Korea. Tel: 82-2-2225-1417, Fax: 82-2-2225-1416, E-mail: medisun74@naver.com

(c) This is an open-access article distributed under the terms of the Creative Commons Attribution Non-Commercial License (http:// creativecommons.org/licenses/by-nc/3.0/), which permits unrestricted non-commercial use, distribution, and reproduction in any medium, provided the original work is properly cited. 


\section{Case Report}

A 79-year-old female patient with weight of $58 \mathrm{~kg}$ and height of $156 \mathrm{~cm}$ visited the hospital for lumbar pain accompanied by referred pain in the left hip and the rear thigh. She was diagnosed as spinal retrolisthesis and stenosis at L 4-5. She admitted to the hospital for discectomy and partial laminectomy. She had no special past history but a thyroid isthmectomy to remove thyroid nodule in 2005 . She was normal in a blood test, a chest X-ray, and a thyroid function test but had RBBB in ECG. In a pulmonary function test, forced vital capacity (FVC) was $1.42 \mathrm{~L}$ (72.3\% of an expected value), forced expiratory volume at 1 second (FEV1) $1.13 \mathrm{~L}$ (71.1\% of an expected value), and FEV1/FVC $79.4 \%$; which were mild restrictive patterns. However, arterial blood gas analysis was normal. In physical examination, we could find a $3 \mathrm{~cm}$ sized scar from the isthmectomy, $2 \mathrm{~cm}$ above the sternal notch and her breathing sound was normal at both lungs. In addition, she did not appeal any symptom related to a respiratory system. Thus, the lumbar surgery was proceeded as scheduled.

Patient did not receive any preanesthetic medication and she inhaled $100 \%$ oxygen sufficiently after standard monitoring with ECG, non-invasive blood pressure monitor and a pulse oximetry. General anesthesia was induced with lidocaine 40 $\mathrm{mg}$ and propofol $80 \mathrm{mg}$, and rocuronium $40 \mathrm{mg}$ was injected for muscle relaxation after eyelid reflex disappeared. An armored endotracheal tube of $7.0 \mathrm{~mm}$ was inserted and fixed at $21 \mathrm{~cm}$. Breathing sounds at both lung fields were normal. Ventilator was adjusted at $550 \mathrm{ml}$ of tidal volume and 10 times per minute of the respiratory rate. Peak airway pressure was $18 \mathrm{mmHg}$. Anesthesia was maintained with sevoflurane $1.5-2.0$ vol\% in a $50 \%$ of oxygen-nitrous oxide balance. End tidal carbon dioxide tension was maintained at $30-35 \mathrm{mmHg}$. To monitor invasive blood pressure, a $20 \mathrm{G}$ catheter was introduced into the right radial artery and a foley catheter was inserted. For a surgery, the patient changed her position from supine to prone. We check her airway was adequately maintained and her breathing sound normal. Then surgeon began disinfecting around the operation site. While arranging her head on a head rest, the peak airway pressure surged into 35 to $40 \mathrm{mmHg}$, the tidal volume reduced to 50 to $100 \mathrm{ml}$, and her breathing sounds were rarely heard from both lung fields. The endotracheal tube was fixed at 21 $\mathrm{cm}$ as same at the supine position. There was resistance when we tried to insert a suction tube to suck up the endotracheal tube into which the suction tube could not move. We observed the endotracheal tube with a flexible fiberoptic bronchoscopy and reddish soft tissue was almost obstructing the tube (Fig. 1). Although we moved the tube back up to $19 \mathrm{~cm}$ while observing it with the bronchoscopy, the airway obstruction continued. When we moved it further up to $23 \mathrm{~cm}$ again, the obstruction reduced a little bit. As we pushed the front neck lightly with hand, the obstruction was almost eliminated but not completely. Thus we decided to change the patient's position to supine. We laid down the patient on the back and inspected carefully her neck and chest areas. The skin right up the part where the right clavicle and the sternum meet together was somewhat protruded, and the pulse was felt behind the clavicle with hands. When we observed it in the supine position with the bronchoscopy, the internal lumen was maintained at the depth of $23 \mathrm{~cm}$ of the endotracheal tube. The tube was located above the carina and it was not one lung ventilation. At this time, the tidal volume was $500 \mathrm{ml}$ and the peak airway pressure was 18 $\mathrm{mmHg}$ at the respiratory rate of 12 times per minute. Position change to prone allowed ventilation but not as good as in the supine position. Supporting the patient's neck with a cotton roll helped to keep the tidal volume at $500 \mathrm{ml}$, the peak airway pressure at $22 \mathrm{mmHg}$ and normal breathing sounds. After confirming with the fiberoptic bronchoscopy that the airway was obtained and the tip of the tube is located above the carina, the operation was continued. There was no airway obstruction during the operation after that. The operation was completed without any special problem. After we confirmed that the patient was recovered with consciousness and spontaneous breathing, the endotracheal tube was removed. The patient was moved to a ward as she had no problem in recovery. Computed tomography on the neck and the chest showed that the trachea was deviated to the downward right side from the thoracic inlet at which a tortuous innominate artery contact with the trachea
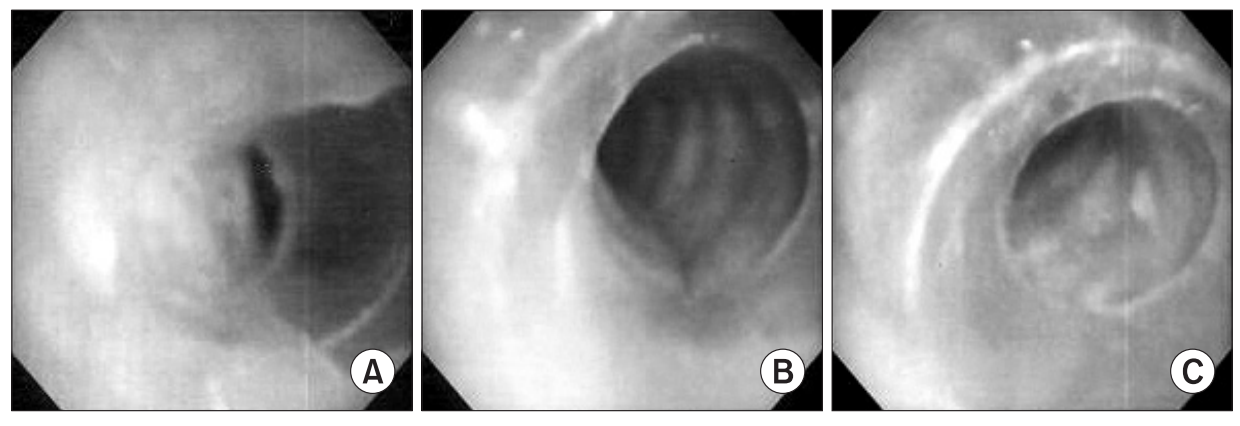

Fig. 1. Bronchoscopic findings of endotracheal tube. (A) Endotracheal tube fixed at $21 \mathrm{~cm}$ is near totally obstructed by soft tissue under prone position. (B) Tracheal lumen is maintained under prone position. The tube is fixed at 23 $\mathrm{cm}$ and the patient's neck is supported by a cotton roll. (C) Tracheal lumen is intact under supine position. The tube is fixed at $23 \mathrm{~cm}$. 

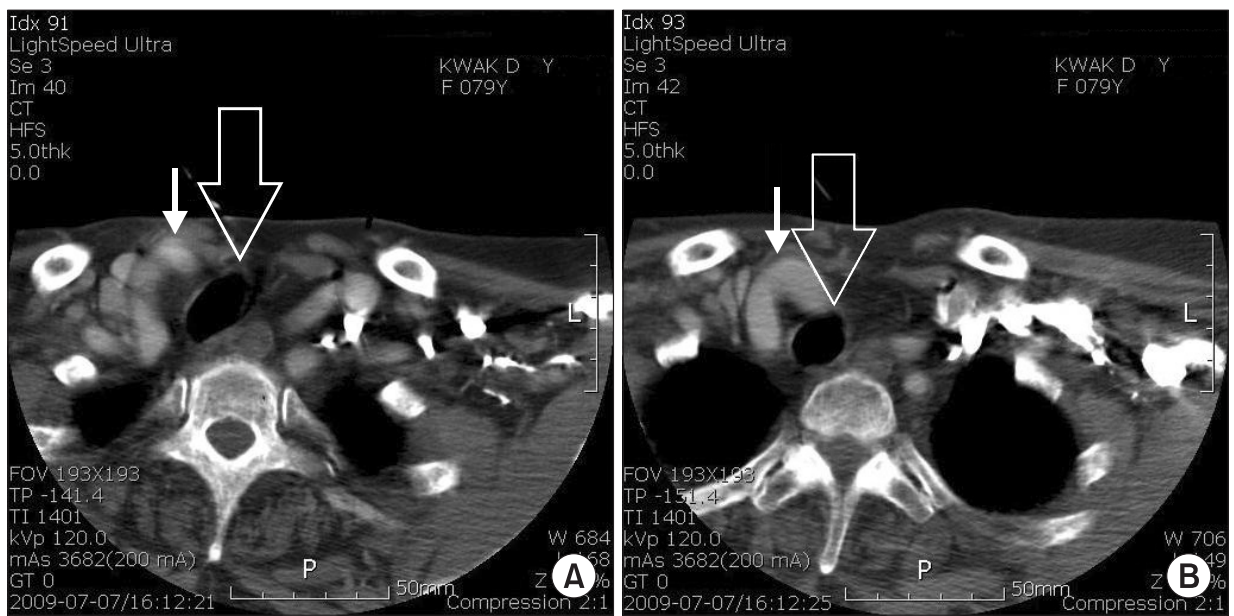

Fig. 2. Neck computed tomography shows rightward tracheal deviation (wide arrow) and tortuous innominate artery (narrow arrow) contact with trachea at right supraclavicular area beneath subcutaneous layer (A), and at thoracic inlet level (B).

(Fig. 2). Beneath the subcutaneous layer of the right clavicle, the distal innominate artery was met the trachea as twisted. In the computed tomography measurement, the innominate artery contact with the trachea in the depth of $19.6 \mathrm{~cm}$ to 21.3 $\mathrm{cm}$ along the trachea from the upper central incisor. When we checked the chest X-ray backward, which was taken at the preoperative examination, it was normal without lesion in lung parenchyma but the trachea was deviated to the right side from the thoracic inlet. As the patient has had no subjective symptom related to vascular problems, she did not take any action against it. The patient was discharged after being noticed that she needs to talk about the history to an anesthesiologist in case of general anesthesia in the future.

\section{Discussion}

Vascular malformation that obstructs the airway occurs relatively commonly in infants and children. In a serious case, it can be corrected by operation in the age of infants or children. Thus, it is rarely found in an adult. In general, it companies with symptoms like respiratory difficulty in exercise, repeated pneumonia, bronchitis, continued stridor in the upper airway, and dysphasia when the vessel compresses the esophagus together. It is often misdiagnosed as asthma in adults [3]. If the innominate artery originates in the distal aortic arch, the artery should turn around the front of the trachea when going to the right upper quadrant to reach to the right thracic apex. When the artery is big and tight, it gives more pressure on the trachea [4]. However, there is a dispute on the classification of the anterior tracheal compression by innominate artery into one of vascular malformation that causes airway obstruction [5]. In the past, there were excessive diagnoses and treatment of innominate artery compression and tracheomalacia was regarded as a major cause of pathophysiology rather than external pressure [6]. In addition, most normal infants had compression on anterior tracheal without respiratory symptoms when the innominate artery came across the trachea in radiologic examinations [7].

It is relatively easy to assess whether the innominate artery compresses the trachea or not. In a simple chest posteroanterior X-ray view, there is no special problem. Meanwhile the lower part of the trachea is narrow and a finger pressed trace is found in the tracheal anterior wall in the lateral view, the posterior wall is right and well arranged [8]. Moreover the simple X-ray helps us to distinguish whether the narrow trachea is caused by underdevelopment of tracheal cartilage by vascular malformations or by simple external compression. If it is caused by external compression, the narrow part is relatively constantly kept as narrow from the beginning to the end of a respiratory period. If the tracheal cartilage matters, a tracheal diameter increases in inspiration and decreases in expiration. Computed tomography is useful to indentify the range of the narrowed area and the degree of obstruction, a change of a tracheal diameter and a location of the tracheal cartilage defect. As the magnetic resonance imaging is non-invasive and shows blood vessels, the heart, tracheobronchial tree, both systemic circulation system and the pulmonary circulation system, recently it is selected as a primary test of all types of vascular compression syndromes [9]. A structure of the tracheobronchial tree can be observed with a bronchoscopy. It makes possible to assess functional factors of airway obstruction or intrinsic lesion such as tracheomalacia, tracheal stenosis, total tracheal cartilage ring, tracheal variation, as well as to know the location of an endotracheal tube in general anesthesia. We can determine the proper size and the depth of the endotracheal tube by evaluating the location and length of the compressed area and the degree of obstruction.

In anesthesia of a patient with airway obstruction by vascular compressions, it is helpful not to use muscle relaxants while keeping self-respiration for maintaining the airway [1]. 
Although some forced expiration can prevent airway from being obstructed during the exhalation, excessive efforts during expiration or respiration in an unstable patient can aggravate tracheal obstruction in the dynamic intrathoracic cavity and further increase turbulent flow around the obstructed area. Thus, it is important to understand a cause, a degree and a location of the damaged airway. When the trachea became too much narrow, the endotracheal tube should be located neighboring the lesion passing the narrow part to prevent the formation of edema or granulation tissue due to the tube. It is safe to insert a stent using a rigid bronchoscope or an endotracheal tube to obtain proper ventilation in a patient with serious tracheal collapse.

In the case of the study, we considered correlation with a thyroid isthmectomy at the very beginning because the patient has a history of the operation. However, the operation scar at $2 \mathrm{~cm}$ upward from the sternal notch was distant from the area where the right clavicle and the sternum meet, at which pulse is felt. In addition, as the isthmectomy is to remove isthmus only while preserving the both thyroid lobes, it is too minimal invasive to cause complications such as adhesion or hematoma. Although the tracheal cartilage defect could not be completely excluded, tracheomalacia or cartilage defect was not considered as a cause because the patient had no complaint on the respiratory system. Airway obstruction by external compression is not common in adults whose trachea is completely matured. However, there is a case that adult male patient had severe tracheal obstruction due to the pseudomeningocele after induction of general anesthesia. Thus we assumed that the airway obstruction in our patient was caused by external compression by blood vessels [10]. In this case, the blood vessel does not encircle the trachea like a ring and the innominate artery is branched in the normal location of aortic arch. Although the innominate artery is tortuous and goes in a bended shape, it does not compress the trachea directly and the patient has had no respiratory symptom. Therefore it is reasonable to see the innominate artery as normal variation rather than malformation. When the patient changed the position into prone, the chest was compressed and thus innominate artery contact with the trachea compressed the trachea. As the endotracheal tube was inserted toward the carina, the tip of the tube might pass the compressed area and maintain the endotracheal lumen. In addition, we guess that the cotton roll supporting the patient's neck in the prone position pushed the trachea backward and released the anterior tracheal compression by the tortuous artery at the thoracic inlet level to some extent. As the patient was old and had no respiratory problem for a life, we discharged her without special treatment. Also, we recommended her to talk about the anesthesia-related history to an anesthesiologist in the future if it is necessary to intubate an endotracheal tube with general anesthesia because the tortuous innominate artery and the rightward deviated trachea can cause the tube obstruction again.

As the airway obstruction by vascular malformations is rarely found, it can be misdiagnosed as a respiratory disease such as asthma when only respiratory symptoms occur. Anesthesioloists should understand mechanical features of airway obstruction by vascular malformations in advance when conducting general anesthesia for a patient who has a respiratory symptom due to unidentified airway obstruction in order to make a diagnosis and treatment in a more accurate and rapid manner.

\section{References}

1. Kussman BD, Geva T, McGowan FX. Cardiovascular causes of airway compression. Paediatr Anaesth 2004; 14: 60-74.

2. Bose S, Hurst TS, Cockcroft DW. Right-sided aortic arch presenting as refractory intraoperative and postoperative wheezing. Chest 1991; 99: 1308-10.

3. Hardin RE, Brevetti GR, Sanusi M, Bhaskaran D, Burack JH, Genovesi MH, et al. Treatment of symptomatic vascular rings in the elderly. Tex Heart Inst J 2005; 32: 411-5.

4. Myer CM 3rd, Wiatrak BJ, Cotton RT, Bove KE, Bailey WW. Innominate artery compression of the trachea: current concepts. Laryngoscope 1989; 99: 1030-4.

5. McLaren CA, Elliott MJ, Roebuck DJ. Vascular compression of the airway in children. Paediatr Respir Rev 2008; 9: 85-94.

6. Mahboubi S, Gheyi V. MR imaging of airway obstruction in infants and children. Int J Pediatr Otorhinolaryngol 2001; 57: 219-27.

7. Strife JL, Baumel AS, Dunbar JS. Tracheal compression by the innominate artery in infancy and childhood. Radiology 1981; 139: 73-5.

8. Gross RE. Arterial malformations which cause compression of the trachea or esophagus. Circulation 1955; 11: 124-34.

9. van Son JA, Julsrud PR, Hagler DJ, Sim EK, Puga FJ, Schaff HV, et al. Imaging strategies for vascular rings. Ann Thorac Surg 1994; 57: 604-10.

10. Blank RS, Waldrop CS, Balestrieri PJ. Pseudomeningocele: an unusual cause of intraoperative tracheal compression and expiratory obstruction. Anesth Analg 2008; 107: 226-8. 